Methods As a retrospective review, we examined 299 patients with 339 aneurysms, all completely occluded at 36 months in follow-up images obtained between 2011 and 2013. Medical records and radiologic data acquired during extended monitoring (mean, $74.3 \pm 22.5$ months) were retrieved, analyzing incidence (including average annual risk) and risk factors of delayed recanalization.

Results A total of five coiled aneurysms (1.5\%) occluded completely at 36 months showed recanalization $(0.46 \%$ per aneurysm-year) during continued long-term surveillance (1081.9 aneurysm-years), two surfacing within 60 months and three developing thereafter. Four showed minor recanalization, with only one instance of major recanalization. The latter involved posterior communicating artery as an apparent de novo lesion, arising at the neck of a firmly coiled sac, and was unrelated to coil compaction or growth. Additional embolization was undertaken. In multivariate analysis, second embolization for recurred aneurysm $(\mathrm{HR}=22.088, \mathrm{p}=0.003)$ independently correlated with delayed recanalization.

Conclusion Almost all coiled aneurysms (98.5\%) showing complete occlusion at 36 months post-embolization proved to be stable in extended observation. Therefore, it is reasonable to suspend imaging surveillance of coiled aneurysms after 3 years in the absence of demonstrable recanalization. However, recurrent aneurysms were predisposed to delayed recanalization. Disclosures Y. Cho: None.

\section{P-036 NOVEL LIQUID EMBOLIC MATERIAL FOR THE USE OF ENDOVASCULAR TREATMENT: A HYDROPHILIC POLYMER COMPOSITE ACTIVATED BY THE CA2+ IN THE BLOOD}

${ }^{1}$ Y Yuki ${ }^{*},{ }^{2} \mathrm{~K}$ Ohkawa, ${ }^{1} \mathrm{~F}$ Hsu, ${ }^{1} \mathrm{~S}$ Suzuki. ${ }^{1}$ Neurosurgerey, UC Irvine Medical Center, Orange, $C A ;{ }^{2}$ Bioengineering, Institute for Fiber Engineering Shinshu University, Ueda, Japan

\subsection{6/neurintsurg-2019-SNIS.72}

Introduction/Purpose The New Generation Liquid Embolic Material (NGLEM) is a clear liquid that immediately forms a solid hydrogel cast upon exposure to $\mathrm{Ca} 2+$ in the bloodstream. Catheter entrapment is avoided because the material is not adhesive, and the use of organic solvents such as DMSO is unnecessary. The main components of NGLEM are widely used as food additives in the medical and food industries, with much evidence of their biocompatibility. The mechanical behavior of this new liquid embolic material was evaluated using an in vitro vascular model as well as an in vivo experimental model using a swine.

Materials and methods Experiment 1) A silicon vascular model with multiple aneurysms were connected to a programmable pulsatile flow pump, and isotonic aqueous solution containing $2 \mathrm{mM}$ calcium chloride was circulated. A microcatheter was placed in the aneurysm model, and $1 \mathrm{ml}$ of NGLEM was injected. The behaviors of the injected material and the visibility under fluoroscopy were recorded. Experiment 2) To evaluate the mechanical performance of the NGLEM using in vivo model, a rat renal artery was catheterized with a microcatheter, and NGLEM was injected into the artery. Angiographical evaluation was performed.

Results Experiment 1) The injected NGLEM formed spherical shaped gel and occluded the aneurysm. Once the injection was completed, the catheter was withdrawn without entrapment. The NGLEM mixed with contrast medium showed sufficient radiopacity under fluoroscopy. Experiment 2) A total of 5 renal arteries of 3 rats were treated with NGLEM. All procedure was performed without technical complications. All vessels were completely occluded with NGLEM, and the total volume required for the occlusion was between $0.4 \mathrm{ml}$ to 0.8 $\mathrm{ml}$. No increased thrombogenicity was observed during the procedure. Post procedure aortogram showed complete occlusion of the treated artery.

Conclusion NGLEM, which is a DMSO free, non-adhesive bio-polymer may be used as an embolic material for endovascular procedure.

Disclosures I. Yuki: 1; C; Institute for Clinical and Translational Science (ICTS) Pilot Award (NIH Director's Transformative Research Award initiative), Principle Investigator, April 1st. 2019 - present. K. Ohkawa: 1; C; 17H02080, Japan Society for the Promotion of Science Ohkawa, Kousaku (PI) 04/01/17-03/31/21. F. Hsu: 1; C; Institute for Clinical and Translational Science (ICTS) Pilot Award (NIH Director's Transformative Research Award initiative). S. Suzuki: 1; C; Institute for Clinical and Translational Science (ICTS) Pilot Award (NIH Director's Transformative Research Award initiative).

Conclusion NGLEM, which is a DMSO free, non-adhesive bio-polymer may be used as an embolic material for endovascular procedure.

Disclosures I. Yuki: 1; C; Institute for Clinical and Translational Science (ICTS) Pilot Award (NIH Director's

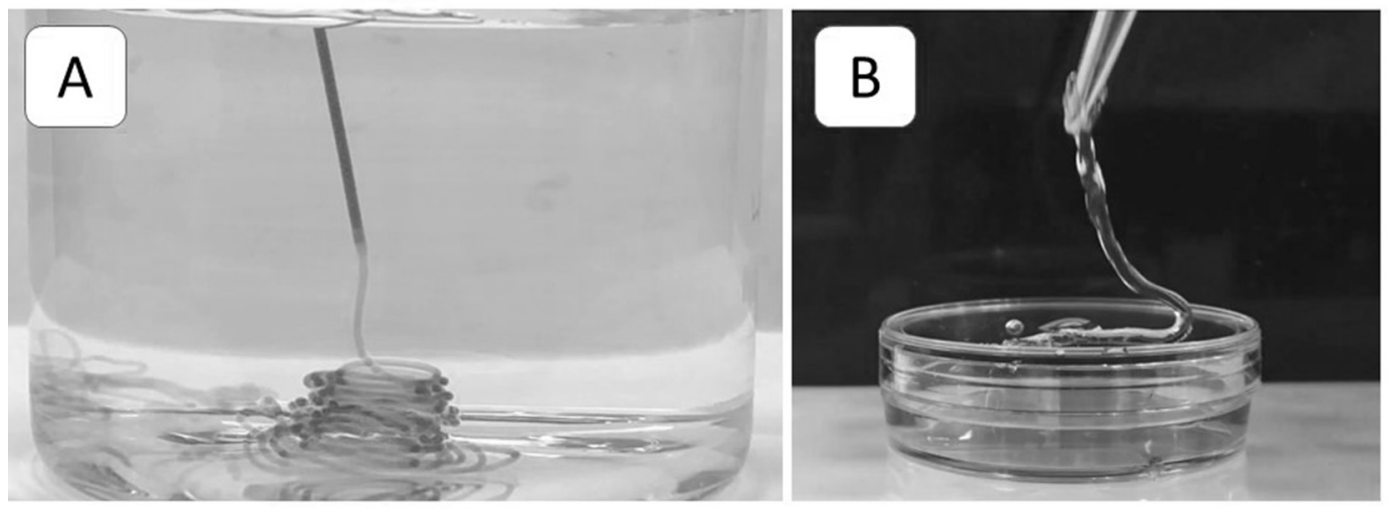

Abstract P-036 Figure 1 NGLEM injection in a static electrolyte solution (A) The injected NGLEM forms a cylindrical shaped hydrogel upon exposure to a buffered electrolyte solution. (B) A segment of the hydrogel is extracted from the solution, and its mechanical strength is tested. Note the hydrogel picked up and held with a forceps shows the rubberlike property without being torn off by a vigorous shake. 
Transformative Research Award initiative), Principle Investigator, April 1st. 2019 - present. K. Ohkawa: 1; C; 17H02080, Japan Society for the Promotion of Science Ohkawa, Kousaku (PI) 04/01/17-03/31/21. F. Hsu: 1; C; Institute for Clinical and Translational Science (ICTS) Pilot Award (NIH Director's Transformative Research Award initiative). S. Suzuki: 1; C; Institute for Clinical and Translational Science (ICTS) Pilot Award (NIH Director's Transformative Research Award initiative).

\section{P-037 DEVELOPMENT OF A PROTEOMIC BIOMARKER SIGNATURE FOR IDENTIFYING INTRACRANIAL ANEURYSMS}

D Nistal ${ }^{*}$, M Martini, T Hardigan, R Song, J Mocco, C Kellner. Neurosurgery, Icahn School of Medicine, New York, NY

\subsection{6/neurintsurg-2019-SNIS.73}

Introduction Intracranial aneurysms occur in approximately 1$2 \%$ of the general population and are the leading cause for subarachnoid hemorrhage (SAH). Recent studies have shown the utility of using multiplex immunoassays in biomarker discovery for a variety of diseases. Although a number of biomarkers have been identified to be related to intracranial aneurysms, a proteomic signature has not been defined to predict the presence of an intracranial aneurysm. In this study, we utilized proteomic data from patients with known intracranial aneurysms and healthy controls to identify a proteomic signature in the serum to predict the presence of an aneurysm.

Methods Fifty-six patients were prospectively enrolled in this study, 28 of which with unruptured intracranial aneurysms and 28 sex, age, and comorbidity matched healthy controls. Serum was collected from each patient. Protein expression levels were determined using the Proseek multiplex immunoassay (http://www.olink.com/), which included 92 known inflammatory markers. Univariate and multivariate logistic regression models were constructed and systematically assessed using statistical and biological inferences to identify phenotypic and proteomic variables that significantly predicted presence of an aneurysm.

Results Of the 28 patients with intracranial aneurysms, $82.1 \%$ $(n=23)$ were female, $46.4 \% \quad(n=13)$ were never smokers, $35.7 \%(n=10)$ were former smokers, and $17.9 \%(n=5)$ were current smokers. Healthy controls were matched on a 1:1 basis, with age \pm 5 years. In the patient cohort the mean aneurysm size was $8.9 \mathrm{~mm}$ with the most common location being anterior communicating artery $(35.7 \%, n=10)$. Upon univariate analysis, increased expression of 10 analytes were found to be independent predictors of the presence of an intracranial aneurysm. At the univariate level, IL17 $(\mathrm{OR}=12.01 ; 95 \%$ CI $4.16-$ 53.17; $\mathrm{p}<0.00001)$ and $\mathrm{CD} 244(\mathrm{OR}=34.19 ; 95 \%$ CI 6.14 $344.81 ; \mathrm{p}=0.000531)$ were found to be the most predictive. At the multivariate level, while controlling for age, sex, hypertension, and smoking status, IL17 remained a significant predictor of the presence of an intracranial aneurysm $(\mathrm{OR}=32.70$, 95\% CI 4.64 - 778.81; $\mathrm{p}=0.00485)$.

Conclusion This study leveraged individualized data from patients with intracranial aneurysms to determine which proteomic variables predict the presence of an intracranial aneurysm while controlling for essential clinical covariates. Larger multi-omic studies are warranted to develop a more comprehensive biomarker signature for intracranial aneurysms.
Disclosures D. Nistal: None. M. Martini: None. T. Hardigan: None. R. Song: None. J. Mocco: 1; C; Penumbra Inc.. 2; C; Cerebrotech, Rebound Therapeutics, TSP Inc, Lazarus Effect, Medina, Pulse Vascular. C. Kellner: 1; C; Siemens, Penumbra, Bee Foundation.

\section{P-038 PREDICTORS OF VENTRICULOSTOMY INFECTION IN A LARGE COHORT}

A Sweid*, S Tjoumakaris, C Wamsley, E Mann, C Neely, J Head, M Gooch, N Herial, T Alexander, S Missios, N Chalouhi, R Rosenwasser, P Jabbour. Neurosurgery, Thomas Jefferson University, Philadelphia, PA

10.1136/neurintsurg-2019-SNIS.74

Background External ventricular drain (EVD) placement is a common neurosurgical procedure. While this procedure is simple and effective, infection is a major limiting factor. Factors predictive of infection reported in the literature are not conclusive.

Objective The aim of this retrospective single-center large sample was to assess the rate and factors predictive of infection.

Methods We performed a retrospective review of patients who underwent EVD placement between January 2012 and January 2018.

Results A total of 714 patients were included in the study. The infection rate was $3.6 \%(26 / 714)$. In $45 \%$ of the cases, the indication for ventriculostomy placement was for acute subarachnoid hemorrhage (aSAH) or intracerebral hemorrhage (ICH). Of the factors that were an independently significant predictor of ventriculostomy-associated infection (VAI) was the placement of a second EVD; the likelihood was eight times higher than if only a single EVD was placed (OR: 7.95, 95\% CI: 1.01-62.9, p<0.05). Another factor was routine CSF sampling, associated with greater than nine times higher likelihood of VAI compared to no routine CSF sampling (OR: 9.20, 95\% CI: 1.93-43.9, p<0.01). Intrathecal (IT) drug deliveries were also associated with a higher likelihood of VAI (OR: 55.7, 95\%CI: 2.92-

1060, $\mathrm{p}<0.01)$. Non-significant factors were day vs. night, location (ICU/ER vs. OR), duration, CSF leak, suture type, system flushing.

Conclusion Our study shows that the rate of VAI was $3.6 \%$. Only three variables were significant independent predictors of VAI; these were the replacement of the EVD, routine CSF aspiration, and intrathecal drug delivery.

Disclosures A. Sweid: None. S. Tjoumakaris: None. C. Wamsley: None. E. Mann: None. C. Neely: None. J. Head: None. M. Gooch: None. N. Herial: None. T. Alexander: None. S. Missios: None. N. Chalouhi: None. R. Rosenwasser: None. P. Jabbour: None.

\section{P-039 INTRAVASCULAR ULTRASOUND OF FLOW DIVERTERS IN ANEURYSM TREATMENT}

${ }^{1} \mathrm{~A}$ Nicholson*, 'S Taylor, ${ }^{2} \mathrm{C}$ Woods, ${ }^{1} \mathrm{~J}$ Cuoco, ${ }^{1} \mathrm{~B}$ Klein, ${ }^{1} \mathrm{E}$ Guilliams, ${ }^{1} \mathrm{E}$ Marvin, ${ }^{1} \mathrm{~J}$ Entwistle. ${ }^{1}$ Neurosurgery, Carilion Clinic, Roanoke, VA; ${ }^{2}$ Virginia Tech Carilion School of Medicine, Roanoke, VA

10.1136/neurintsurg-2019-SNIS.75

Introduction/Purpose The use of flow diverting stents for the treatment of cerebral aneurysms has significantly increased since the PUFS (Pipeline for Uncoilable of Failed Aneurysms) 